Ann. Biol. anim. Bioch. Biophys., I975, 15 (4), 65I-66o.

\title{
ÉTUDE AUTORADIOGRAPHIQUE DE L'ACÉTYLATION DES PROTÉINES NUCLÉAIRES AU COURS DE LA SPERMIOGENĖSE DU CRIQUET
}

\author{
D. BOUVIER et Ph. CHEVAILLIER \\ Laboratoire de Biologie cellulaire, E. R. A. no 400 , \\ Université Paris - Val de Marne, \\ 94000 Créteil
}

\begin{abstract}
RÉSUMÉ
Une étude autoradiographique, menée en microscopie photonique et électronique, montre que les noyaux des spermatides du Criquet Locusta migratoria incorporent l'acétate- ${ }^{3} \mathrm{H}$, en particulier durant les premiers stades de la spermiogenèse, avec un maximum d'incorporation au stade II. Au cours de ce stade et au stade suivant, le noyau de la spermatide subit de profondes modifications structurales et chimiques : la chromatine se décondense et les histones de type somatique sont progressivement remplacées par des protéines basiques plus riches en arginine. L'incorporation maximum d'acétate coïncide ainsi avec la décondensation de la chromatine et précède le début de la transition histones-protéines basiques enrichies en arginine.

Des contrôles cytochimiques et autoradiographiques ont été réalisés de manière à préciser la localisation de l'acétate- ${ }^{3} \mathrm{H}$ au niveau des différents constituants nucléaires. L'acide trichloracétique a été utilisé à différentes concentrations, ainsi que l'acide chlorhydrique, la pronase et la DNase. Il ressort de ces expériences que les protéines, et parmi celles-ci les histones, sont les seuls constituants nucléaires acétylés au cours de la spermiogenèse. L'acétylation des histones semble donc jouer un rôle important dans la modulation des interactions DNA-histones et le remplacement de ces dernières en fin de spermiogenèse.
\end{abstract}

\section{INTRODUCTION}

Au cours de la spermiogenèse du Criquet, les histones de type somatique associées au DNA nucléaire des jeunes spermatides sont remplacées par des protéines plus riches en arginine et, par suite, plus basiques. Ces variations dans la nature des protéines nucléaires sont rendues possibles par une élimination préalable des histones somatiques. Cette élimination, précédée d'une modification de la structure du noyau [8] doit s'accompagner d'une réduction des forces d'interaction entre le DNA et les histones. De telles modifications ont été observées dans différents 
systèmes cellulaires, en relation avec une acétylation des histones. C'est le cas, par exemple, du foie en régénération, des 1ymphocytes et de nombreux tissus soumis à un stimulus hormonal $\lceil\mathrm{I}]$. Dans tous ces exemples, le taux élevé d'acétylation des histones et la stabilité de l'acétate incorporé sont en rapport avec l'activation de la synthèse de RNA.

Pendant la spermiogenèse, la synthèse de RNA s'annule, mais on peut penser qu'un même mécanisme intervient pour réduire l'affinité des histones pour le DNA et permettre ainsi le remplacement de ces dernières par les protéines spécifiques du spermatozoïde. Ainsi, chez la Truite $[7$, I2 $]$ et chez le Rat [Io], une incorporation d'acétate dans les noyaux du testicule a pu être mise en évidence.

La majorité des études se rapportant à l'acétylation des histones utilisent des techniques biochimiques. Quelques travaux autoradiographiques seulement ont été effectués. MUKHERJEE et COHEN [I3] ont apporté la preuve cytologique d'une relation temporelle entre les incorporations d'acétate et d'uridine dans les lymphocytes humains soumis à l'action de la phytohémagglutinine. Par la mêtme technique, ELLGAARD [9] et AlLFREY et al. [2] ont tenté de localiser 1'incorporation d'acétate radioactif le long des chromosomes géants de la glande salivaire de la Drosophile et du Chironome. Berlowitz et PALLOTTA [3] ont comparé par cette méthode l'aptitude de l'euchromatine et de 1'hétérochromatine à utiliser l'acétyl-Coenzyme $\mathrm{A}-{ }^{3} \mathrm{H}$ comme donneur d'acétate aux histones. L'approche autoradiographique, bien que moins précise que les méthodes biochimiques, nous a permis de localiser dans le déroulement de la spermiogenèse l'incorporation nucléaire d'acétate au niveau des différents types de spermatides. A l'échelle du microscope électronique, cette méthode permet d'autre part de relier l'incorporation d'acétate aux divers aspects ultra-structuraux de la chromatine. Enfin, différents tests ont été effectués dans le but de préciser sous quelle forme se trouvait incorporé ce marqueur radio-actif.

\section{MATÉRIEL, ETT MÉTHODES}

Les jeunes Criquets mâles adultes recoivent chacun une injection intra-abdominale de I $00 \mu \mathrm{Ci}$ d'acétate de sodium- ${ }^{3} \mathrm{H}$ (NEN, $\left.95 \mathrm{mCi} / \mathrm{mmole}\right)$ et sont laissés en contact avec l'isotope pendant des temps variant entre 5 minutes et 6 heures. Dans une autre série d'expériences, l'incorporation a été suivie dans les follicules testiculaires isolés et incubés dans un milieu de culture pour Orthoptères (milieu de Carlson) contenant de l'acétate ${ }^{3} \mathrm{H}$ ì raison de $100 \mu \mathrm{Ci} / \mathrm{ml} \mathrm{de} \mathrm{milieu.}$

Les fragments de testicules prélevés sur les animaux après l'injection ou sortant du milieu d'incubation sont fixés, soit dans le formol neutre à 4 p. Ioo pour l'étude en microscopie photonique, soit dans la glutaraldéhyde à $2,5 \mathrm{p}$. Ioo en tampon phosphate $0, \mathrm{I} M$ à $\mathrm{pH}$ 7,2 pour l'étude en microscopie électronique.

Les coupes de matériel inclus dans la paraffine et les coupes semi-fines sont recouvertes d'une émulsion autoradiographique Kodak AR-Io. Après développement, les coupes de matériel inclus dans la paraffine sont colorées par le Nuclear I ast Red, et les coupes semi-fines par le bleu de méthylène en solution à o.I3 $\mathrm{p}$. Ioo dans un tampon citrate o, IM à pH 4,2. Pour l'autoradiographie en microscopie électronique, l'émulsion Ilford L-4 a été utilisée; les coupes fines sont contrastées après développement par l'acétate d'uranyle et le citrate de plomb.

En microscopie photonique, les résultats sont analysés pour chaque temps de contact ou d'incubation par comptage direct des grains d'argent localisés sur les noyaux des spermatides. Ces valeurs sont résumées par le nombre moyen de grains par noyau ct l'écart-type correspondant.

Des extractions dcs constituants nucléaires ont été réalisées sur coupes et sur matériel non fixé. L'action de l'acide trichloracétique à $5 \mathrm{p}$. Ioo, à $4^{\circ} \mathrm{C}$ et à $90^{\circ} \mathrm{C}, 20 \mathrm{p}$. I 00 à $4^{\circ} \mathrm{C}$, de $1^{\prime}$ acide chlorhydrique $0,25 \mathrm{~N}$, de la pronase et de la DNase a été testéc. 


\section{RÉSULTATS}

Les figures $\mathrm{A}$ et $\mathrm{B}$ résument les deux étapes de l'incorporation d'acétate- ${ }^{3} \mathrm{H}$ dans les noyaux des spermatides : apparition et augmentation du marquage nucléaire pour des temps de contact allant de 5 minutes à deux heures, puis régression générale du marquage pour des incorporations de plus longue durée.

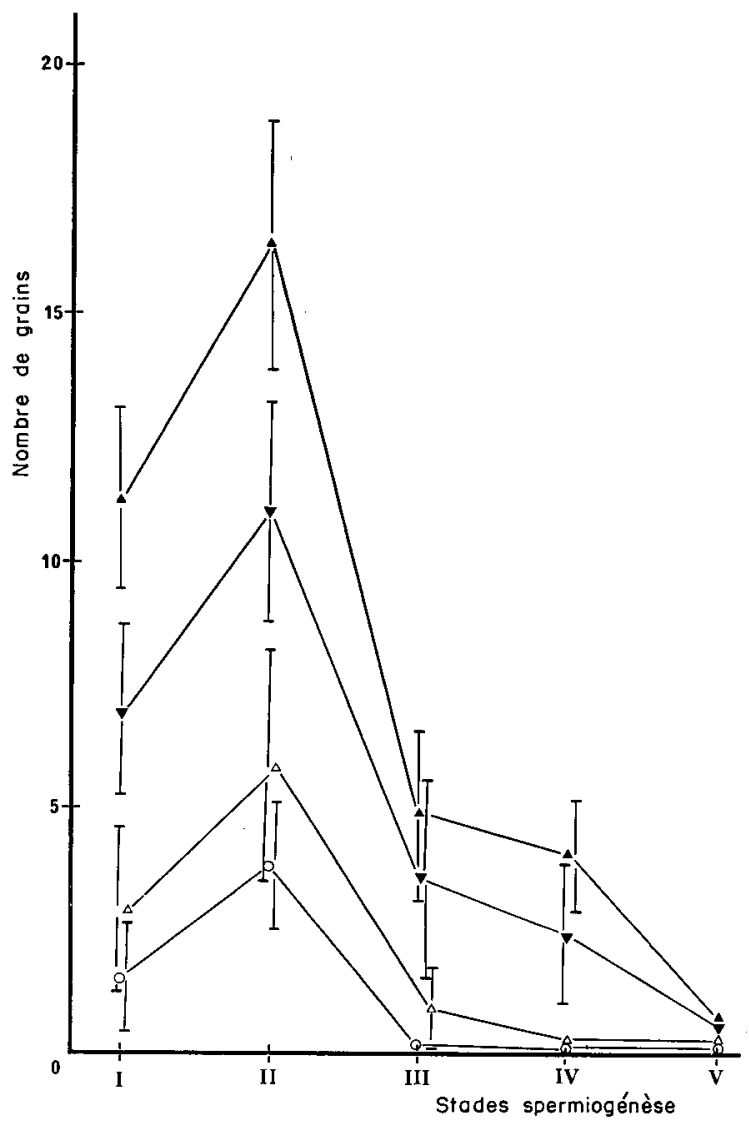

FIG. A. - Incorporation d'acétate dans les noyaux des spermatides du Criquet (Apparition et augmentation du marquage de $5 \mathrm{mn}$ à 2 heures)

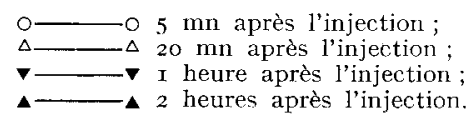

Chez les animaux sacrifiés 5 et $20 \mathrm{mn}$ après l'injection (fig. A), l'acétate est incorporé par les noyaux des premiers stades de la spermiogenèse (stades I et II). Pour des temps de marquage plus longs (I heure, 2 heures), tous les stades sont marqués (fig. I à 4). Les noyaux des stades II se marquent plus rapidement que ceux des 
stades I et III ; un marquage nucléaire s'observe en effet sur ces stades dès 5 minutes après le début de l'incubation, alors que celui que l'on note dans les spermatides au stade I est principalement cytoplasmique. Pour toutes les durées d'expériences retenues ce sont toujours les noyaux des stades II qui sont les plus abondamment marqués. A partir de deux heures (fig. B), la radioactivité nucléaire régresse à tous

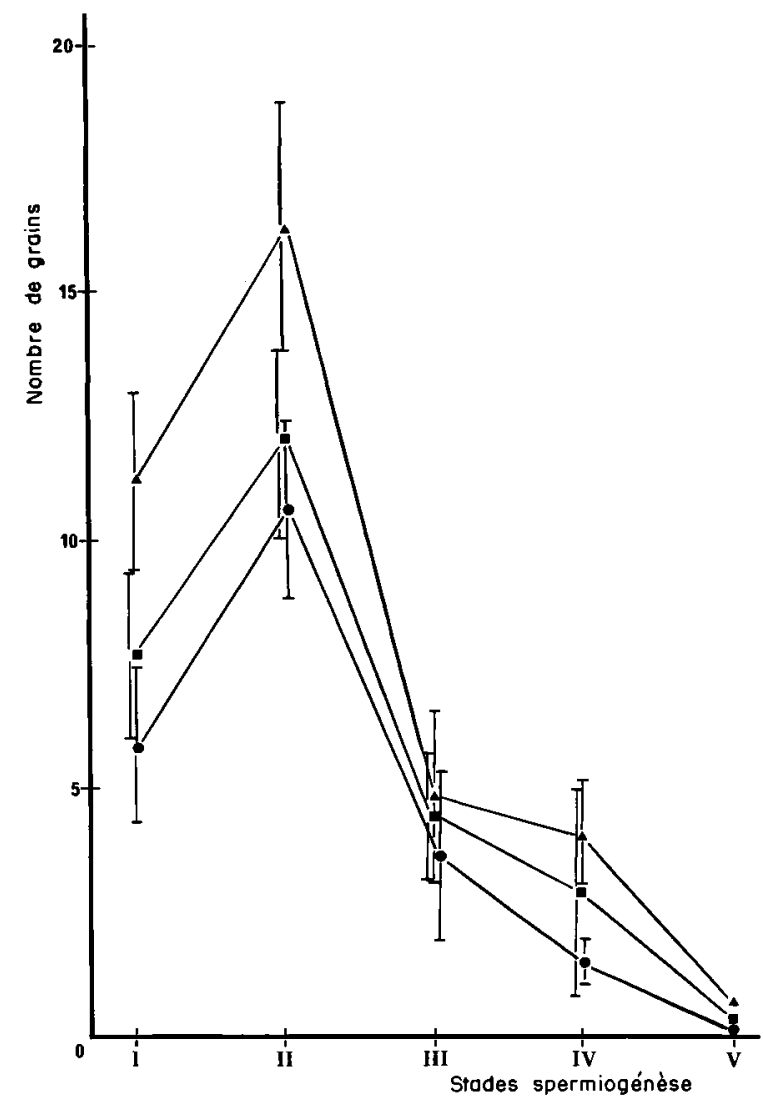

FIG. B. - Incorporation d'acétate dans les noyaux des spermatides du Criquet (Régression du marquage entre deux et six heures de contact)

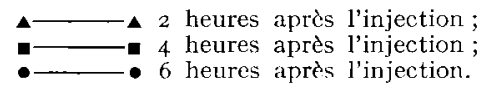

les stades de la spermiogenèse et décrô̂t régulièrement dans le temps. Quatre heures (fig. 5 à 8) ou six heures après l'injection d'acétate (fig. 9 à I2), les noyaux des spermatides au stade II demeurent les plus radioactifs. Six heures après l'injection, le marquage nucléaire est pour tous les stades à peu près la moitié de celui enregistré au bout de deux heures. Les images obtenues sur coupes semi-fines (fig. I3 à I6) donnent une idée plus précise de la localisation des marquages et confirment les résultats décrits ci-dessus.

Lorsque les follicules testiculaires isolés de $1^{\prime}$ 'organe sont mis à incuber à $30^{\circ} \mathrm{C}$ dans le milieu de Carlson contenant l'isotope, les marquages obtenus sont beaucoup 
plus intenses qu'après injection à l'animal entier. Comme le montre le tableau I, pour une heure de contact, les comptages obtenus après incubation sont jusqu'à deux fois plus importants que ceux obtenus après injection.

\section{TABLEAU I}

Comparaison des marquages obtenus après injection d'acétate- ${ }^{3} \mathrm{H}$

$(\mathrm{I}$ oo $\mu \mathrm{Ci} / \mathrm{ml}$ ) ou après incubation dans le milieu de Carlson

(traceur dilué à $\mathbf{~} 00 \mu \mathrm{Ci} / \mathrm{ml}$ ) ;

temps de contact avec le marqueur radio-actif : I heure

\begin{tabular}{c|c|c|c|c}
\hline \multirow{2}{*}{ Mode expérimental } & \multicolumn{3}{|c}{ Stades de la spermiogenèse } \\
\cline { 2 - 4 } & I & II & III & IV \\
\hline Injection & $6,9 \pm 1,7$ & $10,9 \pm 2,2$ & $3,5 \pm 2,0$ & $2,4 \pm 1,4$ \\
\hline Incubation & $12,0 \pm 2,1$ & $11,9 \pm 0,4$ & $5,5 \pm 1,1$ & $4,0 \pm 0,4$ \\
\hline
\end{tabular}

Les résultats sont exprimés par le nombre moyen de grains par noyau de chaque stade et l'écarttype correspondant.

Les effets des différents traitements par l'acide trichloracétique sont résumés dans la figure $\mathrm{C}$.

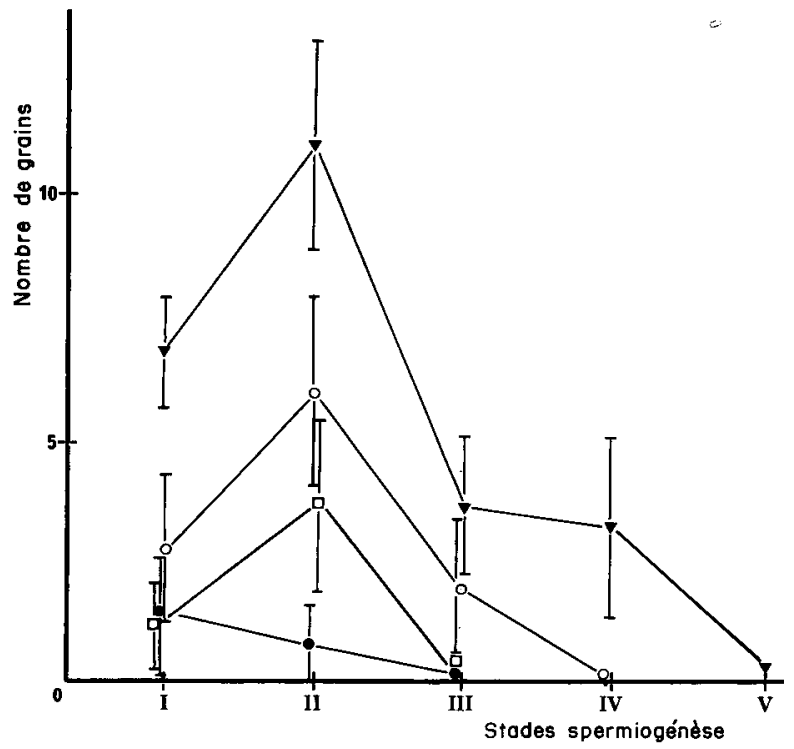

FIG. C. -- Action de l'acide trichloracétique sur un marquage d'une heure par l'acétate

$\longrightarrow$ témoin : marquage d'une heure;

0 - 0 action du TCA à 5 p. roo à $4^{\circ} \mathrm{C}$;

[7-_ـ action du TCA à 5 p. I00 à $90^{\circ \prime} \mathrm{C}$

- - artion de TCA a 20 p. roo à $4^{\circ}$. 
Après extraction de la fraction acido-soluble par l'acide trichloracétique à 5 p. roo à $4^{\circ} \mathrm{C}$ pendant 30 minutes, la moitié de la radioactivité nucléaire de chaque stade est perdue. Dans ces conditions, seuls les noyaux des stades I, II et III demeurent marqués de façon significative (fig. I 7 à I9). L'élimination des acides nucléiques et de la fraction soluble dans 1'acide trichloracétique à 5 p. Ioo, à $90^{\circ} \mathrm{C}$ pendant 15 minutes supprime la radioactivité nucléaire des stades III et IV, ainsi qu'une très forte proportion (environ 70 p. IOO) de celle que l'on détectait dans les stades I et II (fig. 20 et 2I). Le traitement par 1'acide trichloracétique à $20 \mathrm{p}$. Ioo à $4^{\circ} \mathrm{C}$ fait perdre aux noyaux des stades I et II 85 à 90 p. Ioo de l'acétate incorporé (fig. 22 et 23). Le marquage des stades III et IV est totalement effacé. Seuls, les stades I conservent sur leur noyau un marquage significatif.

Les résultats d'autres extractions sont portés sur la figure $\mathrm{D}$. L'extraction des histones par 1'acide chlorhydrique $0,25 \mathrm{~N}$ pendant I heure à $4^{\circ} \mathrm{C}$ fait perdre aux stades I 60 p. Ioo de leur radioactivité nucléaire et aux stades II plus de 90 p. Ioo. I'acétate des stades III et IV est totalement extrait par ce procédé (fig. 28 et 29). La pronase fait perdre aux noyaux de toutes les spermatides la plus grande partie de leur marquage (fig. 30 et $3 \mathrm{I}$ ). Aucune différence significative dans les marquages nucléaires par l'acétate ne peut être remarquée entre les préparations traitées par la DNase et les préparations témoins (fig. 24 à 27).

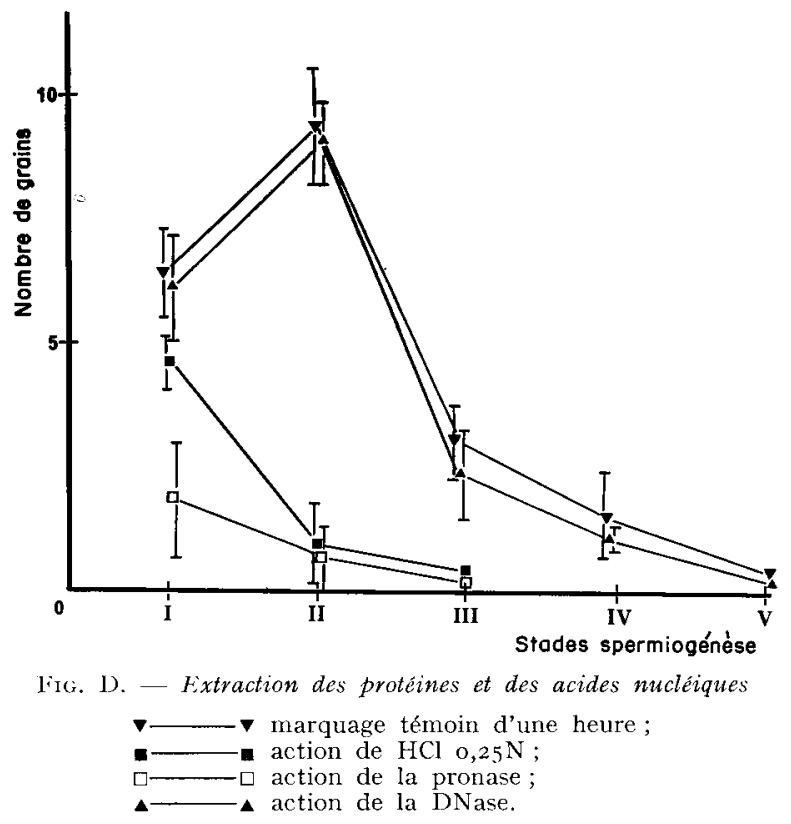

Les résultats obtenus au niveau ultra-structural recoupent les données de l'autoradiographie en microscopie photonique. Une incorporation nucléaire d'acétate a lieu sur les spermatides les plus jeunes (stades I, II et III). Dans les stades plus tardifs, (stades IV et spermatozoïdes) le marquage nucléaire est très réduit ou même nul. I)ans les noyaux des stades I, la radioactivité se détecte principalement sur l'euchro- 
matine. La chromatine décondensée, caractéristique des stades II montre également des grains d'argent (fig. 32) répartis sur la trame fibrillaire des désoxyribonucléoprotéines. Du fait de cette homogénéisation, la localisation du marquage est plus délicate à interpréter. De plus forts grandissements suggèrent que les grains observés pourraient être associés à des régions de chromatine fibrillaire encore partiellement condensées.

\section{DISCUSSION}

Les études cytochimiques et ultra-structurales des noyaux de spermatides du Criquet montrent une relation nette entre les modifications de la composition chimique et de la structure de la chromatine [8]. Parallèlement à ces modifications, on observe des variations de l'incorporation d'acétate dans les noyaux des spermatides au cours de leur différenciation. Chez le Criquet, la chromatine se décondense au stade II, cette décondensation précédant le remplacement des histones par des protéines basiques plus riches en arginine. Ceci s'accompagne d'une forte acétylation des constituants nucléaires. Au contraire, la condensation de la chromatine observée aux stades finaux (stade IV et spermatozoïde) s'accompagne d'une acétylation très réduite puis nulle.

Les résultats préliminaires obtenus après action de l'acide trichloracétique laissaient penser à la présence d'acétate dans de nombreux constituants nucléaires, comme les acides nucléiques ou de petits précurseurs acido-solubles. En fait, parmi les molécules solubles dans l'acide trichloracétique, peuvent se trouver certaines fractions d'histones [6] et des histones acétylées [2]. Les propriétés de solubilité de ces dernières dans les acides sont en effet quelque peu différentes de celles des histones non acétylées, du fait de modifications apportées par l'acétate dans les possibilités d'ionisation de ces protéines basiques. Les histones fortement acétylées (dérivés triou tétraacétyls) sont ainsi solubles dans l'acide trichloracétique à 20 p. IOo [5]. Au contraire, 1'acide picrique conserve ces histones solubles sur les coupes histologiques [4] et permet d'évaluer leur radioactivité due à l'acétate.

Dans les spermatides de stade I, les histones renferment environ $60 \mathrm{p}$. Ioo de l'acétate incorporé par le noyau. Ces résultats sont confirmés par l'extraction des histones dans l'acide chlorhydrique $0,25 \mathrm{~N}$. Dans ces cellules, les histones sont probablement acétylées à un degré relativement faible : 1'acide trichloracétique à $20 \mathrm{p}$. IoO ne modifie pas de façon appréciable la colorabilité des noyaux par les techniques cytochimiques de détection des histones [5]. Par contre, dans les stades II, on trouve des histones fortement acétylées : leur extraction par l'acide trichloracétique à 20 p. Ioo se détecte par voie cytochimique. Ceci peut être mis en relation avec la diminution de leur affinité pour le DNA. La totalité de l'acétate nucléaire est d'ailleurs extraite avec les histones des noyaux de stade II par $\mathrm{HCl} 0,25 \mathrm{~N}$. Les protéines sont sans doute les seuls constituants nucléaires acétylés : 98 à roo p. Ioo de la radio-activité nucléaire est extraite par la pronase.

A l'échelle ultra-structurale, la décondensation de la chromatine au stade II peut être mise en parallèle avec ces phénomènes d'acétylation $[\mathbf{I}]$. Cette décondensation, en modifiant la répartition des fibres de chromatine, permet une meilleure accessibilité de ces dernières aux systèmes enzymatiques d'acétylation. Ceux-ci 
interviennent directement au niveau des complexes nucléoprotéiques [I4], labilisant les interactions DNA-histones et permettant le remplacement des protéines combinées au DNA. I'acétylation ne se traduit pas par des variations du diamètre des nucléoprotéines, mais provoque des modifications très nettes de la stabilité des nucléohistones. Dans les lymphocytes stimulés par la phytohémagglutinine, l'activation de la chromatine, accompagnée d'une forte incorporation d'acétate, se remarque également par une capacité accrue du DNA à fixer l'orangé d'acridine [II] et l'actinomycine $\mathrm{D}[\mathrm{I} 5]$.

Au cours de la spermiogenèse, l'acétylation des histones altère la structure fine de la chromatine, mais ne suffit pas à elle seule à détacher les histones. Elle facilite probablement leur remplacement par d'autres protéines aux stades ultérieurs.

Colloque D. G. R. S. T., Biologie de la Procréation, Paris, $7-8$ mars 1975 .

\title{
REMERCIEMEN'TS
}

Ce travail a été effectué grâce à une aide ̀̀ la recherche de la D. G. R. S. T. (Contrat $n^{0}$ 71-7-3244) et du C. N. R. S. (L. R. A. no 400).

\section{SUMMARY}

\author{
AUTORADIOGRAPHIC STUDY OF NUCL EAR PROTEIN \\ ACETYLATION DURING LOCUST SPERMIOGENESIS
}

\begin{abstract}
Autoradiographic studies, at the light and electron microscope level, demonstrate that spermatid nuclei of the Locust Locusta migratoria incorporate ${ }^{3} \mathrm{H}$-acetate, especially during the first stages of spermiogenesis. The highest level of acetate incorporation is observed during stage II of spermiogenesis. During this stage and the following, the spermatid nucleus undergoes a number of structural and chemical modifications : chromatin decondenses and somatic histones are progressively replaced by newly synthesized arginine-rich proteins. Therefore, the higher degree of acetylation of nuclear components coincides with chromatin decondensation and precedes the protein transition occurring in later stages.

Cytochemical and autoradiographic tests have been realized so as to localize ${ }^{3} \mathrm{H}$-acetate in the nuclear components. Trichloracetic acid was used at various concentrations; the action of hydrochloric acid, pronase and DNase was also tested. The results support the idea that proteins, and among them histones, are the only nuclear components to be acetylated during spermiogenesis. Thus, histone acetylation seems to play an important role in modulating histone-DNA interactions and allowing histone replacement.
\end{abstract}

\section{RÉFÉREINCES BIBLIOGRAPHIQUES}

[1] AllFREY V. G., I968. Structural modifications of chromosomal proteins at times of gene activation. In "Exploitable molecular mechanisms and neoplasia". 22 nd Ann. Symp. on Fundamental Cancer Research. (Baltimore), Williams and Wilkins, ed., 95-rig.

[2] Allfrey V. G., Pogo B. G. T., Littau V. C., Gershey E. L., Mirsky A. E., I968. Histone acetylation in insect chromosomes. Science, 159, 315-3I6.

[3] Berlowitz L., Pallotta D., r972. Acetylation of nuclear proteins in the heterochromatin and euchromatin of mealy bugs. Fexptl. Cell Res., 71, 45-48. 
[4] Bloch D. P., HEw H. Y.C., I96I. Methods for the cytochemical characterization of nuclear basic proteins and their application to problems of development. Ann. Histochimie, 6, 497-500.

15] Bouviti 1)., 1974. Acétylation des protéines nucléaires au cours de la spermiogenèse. Thèse de Doctorat $3^{\mathrm{e}}$ cycle, Paris.

[6] Bustin M., Colf R. D., I968. The applicability of extraction by trichloracetic acid to the preparation of very lysine-rich histones from mammary gland. Arch. Biochem. Biophys., 160, 2 r7-223.

[7] Candido F. P. M., Dixon G. H., I97I. Sites of in vivo acetylation of trout testis histone IV. J. Biochem., 246, 3 182-3188.

[8] Chevallier P., Gusse M., i975. Evolution de la composition chimique et de la structure fine de la chromatine au cours de la spermiogenèse du Criquet Locusta migratoria. J. Micr. Biol. Cell., 23, I $53-\mathrm{I} 64$.

[9] EllgaARd E. G., r967. Gene activation without histone acetylation in Drosophila melanogaster. Science, 157, 1070-1072.

[10] Grimes S. R., Irvin J. L., ChaE C. I3., I973. Acetylation of rat testis nuclear proteins. In Protein phosphorylation in control mechanisms. (Miami Winter Symposia) HUiJing F. et LEE E. Y. C., ed., 5, 321 .

[II] Killander D., Riglek R., I969. Activation of deoxyribonucleoprotein in leukocytes stimulated by phytohemagglutinin. I. Kinetics of the binding of acridine orange to deoxyribonucleoprotein. Expll. Cell Res., 54, 163-170.

[12] Louie A. J., Dixon (x. H., 1972. Synthesis, acetylation and phosphorylation of histone IV and its binding to DNA during spermatogencsis in the trout. Proc. Nat. Acad. Sci., 69, 1975-1979.

[13] Mukhrifee A. B., Cohen M. M., I968. Histone acetylation. Cytological evidence in human lymphocytes. Exptl. Cell Res., 54, 256-260.

[14] Racey L. A., Byvoet P., i97I. Histone acetyltransferase in chromatirl. Extraction of transferase activity from chromatin. Exptl. Cell Res., 73, 329-334.

[15] RINGERTZ N. R., DARZYNKIEWiCz Z., I3OLUND L., I969. Actinomycin Binding properties of stimulated human lymphocytes. Exptl. Cell Res., 56, 4I I-4I6. 


\section{PLANCHE， I}

FIG. I à 4

Marquage observé sur les noyaux de spermatides I heure après l'injection d'acétate sur les noyaux de stade I (fig. I), II (fig. 2), III (fig. 3) et IV (fig. 4).

\section{FIG. 5 à 8}

Marquage observé 4 heures après l'injection d'acétate sur les noyaux de stade I (fig. 5), II (fig. 6), III (fig. 7) et IV (fig. 8).

$$
\text { FIG. } 9 \text { à } \mathbf{I} 2
$$

Marquage observé 6 heures après l'injection d'acétate sur les noyaux de stade I (fig. 9), II (fig. Io), III (fig. II) et IV (fig. I2).

FIG. I3 à 16

Marquages correspondant à I heure de contact sur coupes semi-fines. 

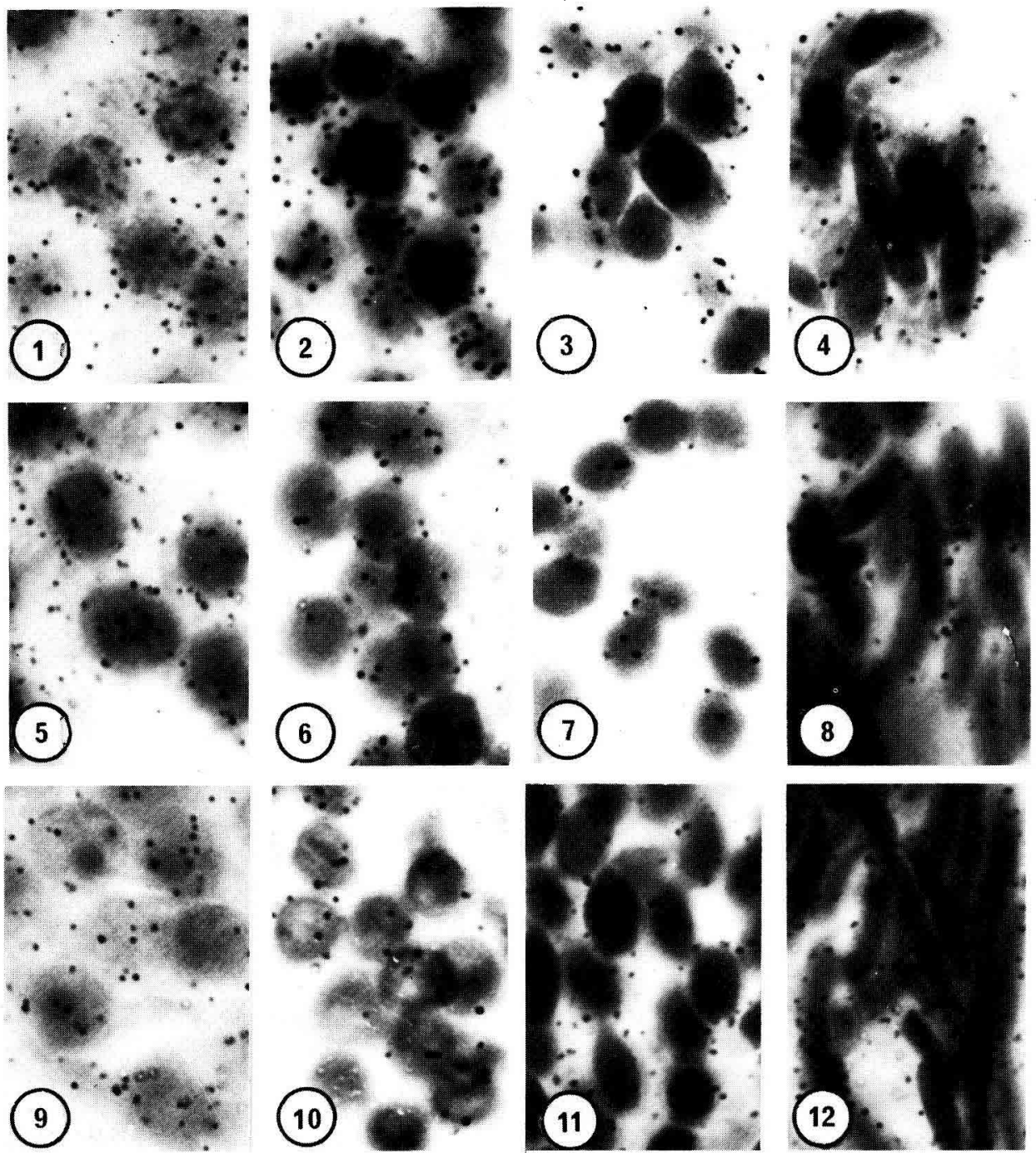

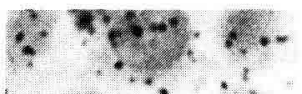

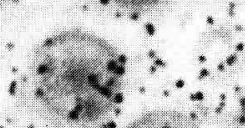
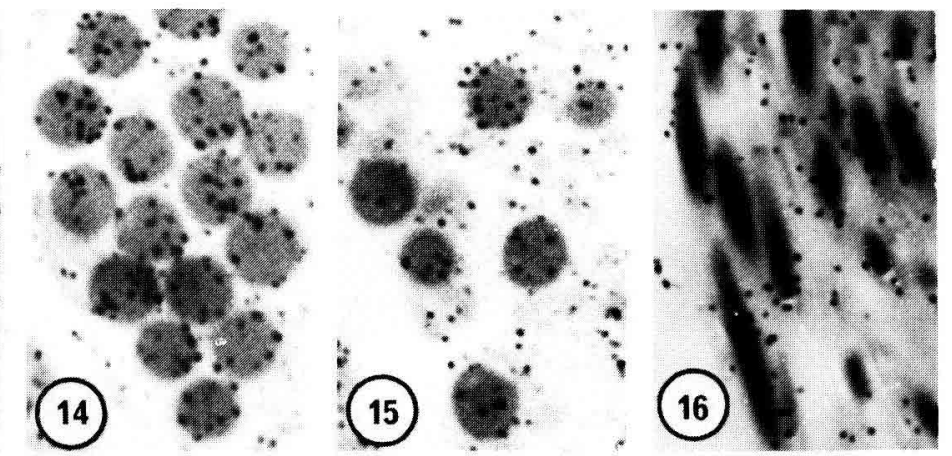

D. BOUVIER et Ph. CHEVAILLIER 


\title{
PLANCHE II
}

\author{
Fig. 17 à 19
}

Action de l'acide trichloracétique à $5 \%$ à $4{ }^{\circ} \mathrm{C}$ sur le marquage des stades I (fig. 17), II (fig. 18) et III (fig. 19).

FIG. 20 et 21

Action de l'acide trichloracétique à $5 \%$ à $90{ }^{\circ} \mathrm{C}$ sur le marquage des stades II et III.

\section{FIG. 22 et 23}

Action de l'acide trichloracétique à $20 \%$ sur le marquage des stades I et II.

\section{FIG. 24 à 27}

Action de la DNase sur le marquage des stades I (fig. 24), II (fig. 25), III (fig. 26) et IV (fig. 27).

FIG. 28 et 29

Action de l'acide chlorhydrique $0,25 \mathrm{~N}$ sur le marquage des stades I et II (observation sur coupes semi-fines).

FIG. 30 et 31

Action de la pronase sur le marquage des stades I et II. 


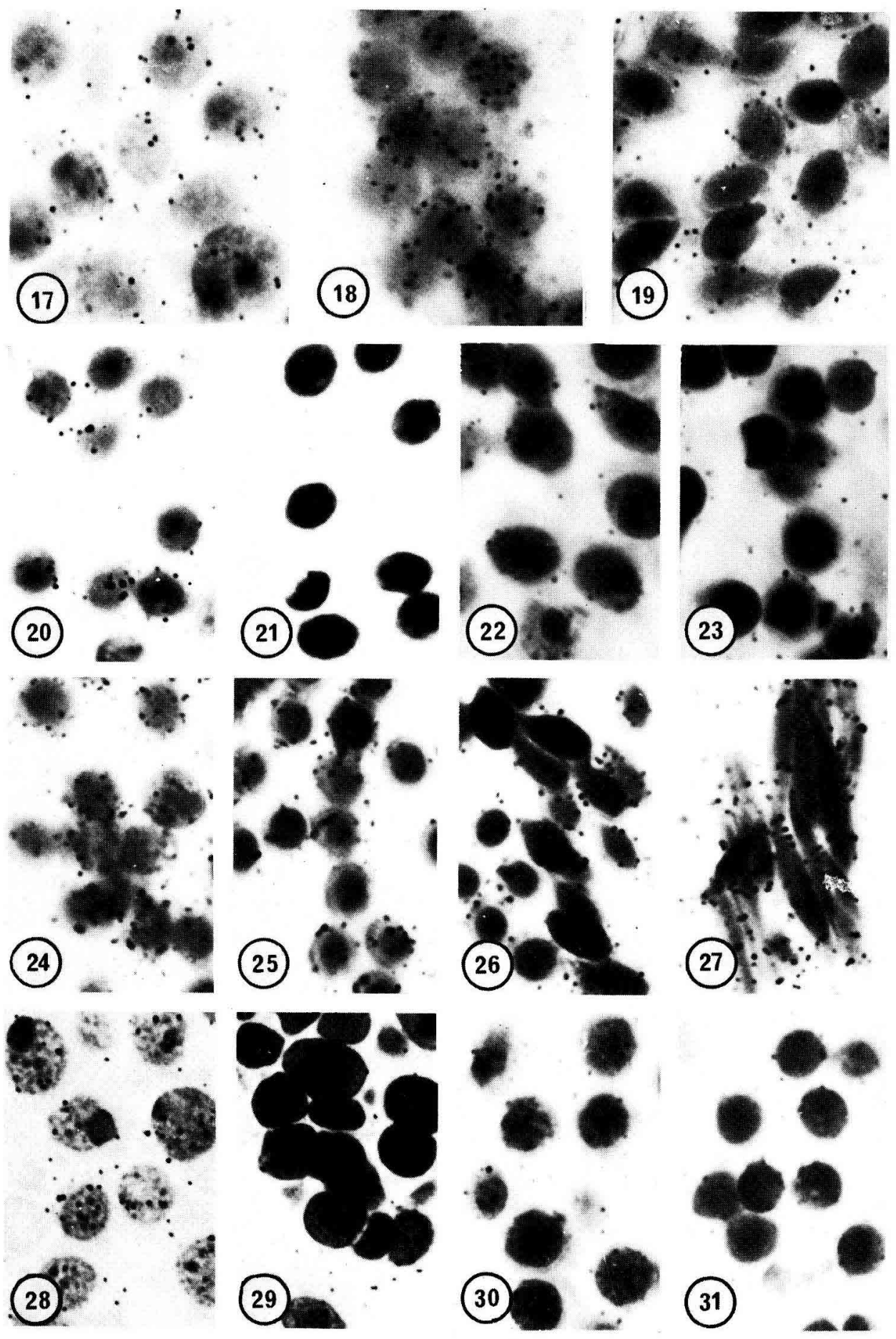

D. Bouvier et Ph. CHEVAILlier 
PLANCHE III

FIG. 32

Marquage observé en microscopie électronique $(\times 14.000)$ sur les noyaux de stade II ( $\mathrm{chr}=$ chromatine ; ce $=$ annexe centriolaire ; $\mathrm{a}=$ acrosome). 

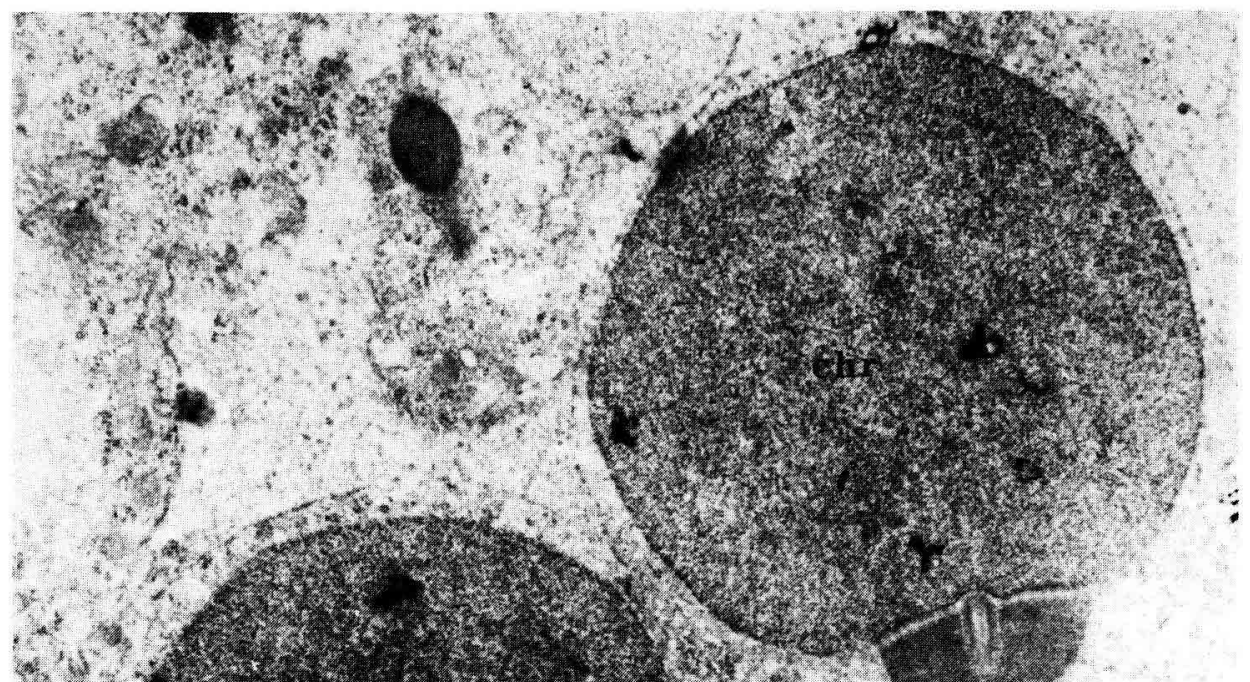

(.) 2,4

$2.3,3^{3}+6$

$5+3+2$

1. 3. $3^{3}+2$

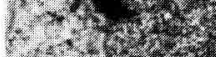

(2: $: 4: 3: 4$

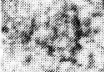

$\therefore-1,20.3$

$34^{2} y^{2}+3$

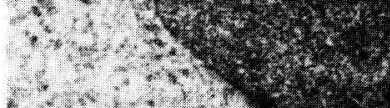

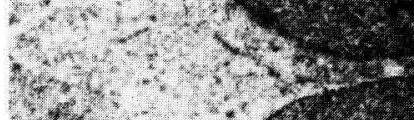

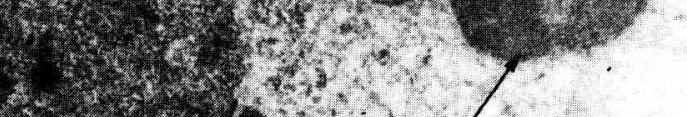

(3)

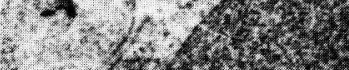

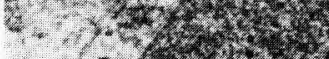

(3) :

6.t.

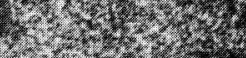

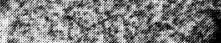

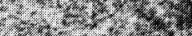

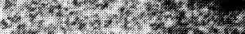

6.2.

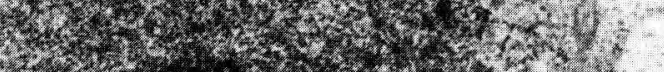

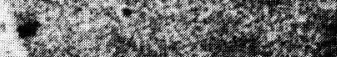

.

-

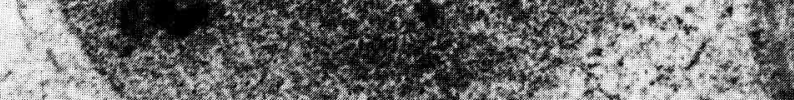

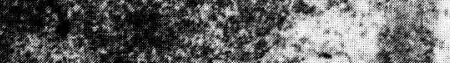

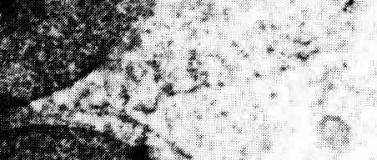

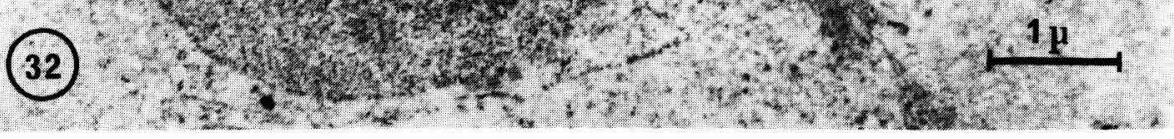

\author{
D. BOUVIER et Ph. CHEVAILLIER
}

